\title{
La formation à l'éthique dans les parcours scientifiques et techniques professionnalisants : lecture croisée des études médicales et d'ingénieurs
}

\author{
Grégory Aiguier ${ }^{1,2, a}$ et Christelle Didier ${ }^{3,4, b}$ \\ ${ }^{1}$ Centre d'Ethique Médicale, Département d'éthique de l'Université Catholique de Lille, 59046 Lille \\ Cedex, France \\ ${ }^{2}$ Laboratoire d'Innovations Pédagogiques, Université Catholique de Lille, 59000 Lille Cedex, France \\ ${ }^{3}$ Laboratoire CIREL équipe Profeor, Université Charles de Gaulle, Lille 3, 59650 Villeneuve d'Ascq, \\ France \\ ${ }^{4}$ Centre d'Ethique Technologique, Département d'éthique de l'Université Catholique de Lille, \\ 59046 Lille Cedex, France
}

\begin{abstract}
Ethics is increasingly considered as a new dimension of professionalism and as a competence professionnals need do develop in order to revisit the foundations, goals and means of their action, driving them to engage in a professional practice that is more critical, reflexive and responsible. The focus on ethics is of particular importance to medicine, but also to other fields of human activities such as engineering where it has benn gaining momentum. The goal of the present study is to present some conceptual developments of a research at thecrossroads of ethics and education, focused on two fields: medicine and engineering.
\end{abstract}

Le contexte de professionnalisation de la médecine fait émerger un nouveau besoin d'éthique. Considérée de plus en plus comme une dimension intrinsèque du professionnalisme (Legault, 2004), l'éthique s'apparente dans ce cadre à une compétence que les professionnels doivent développer pour revisiter les fondements, les finalités, et les moyens de leur agir, et ainsi s'engager dans une pratique professionnelle plus critique, plus réflexive et plus responsable. Sur le plan théorique, cette approche de l'éthique comme compétence, c'est-à-dire comme capacité des acteurs d'initier de nouvelles pratiques en situation et non plus comme discours sur les pratiques, est caractéristique du tournant pragmatique de l'éthique (Maesschalck, 2010). Le souci de l'éthique qui est également présent dans d'autres champs professionnels comme celui de l'ingénierie connaît aussi des évolutions, mais elles ne sont pas du même ordre. Le tournant pragmatique de l'éthique semble requérir une contextualisation plus prononcée de l'apprentissage [situated learning], une articulation plus forte entre théorie et pratique et donc une implication plus grande des terrains professionnels dans la formation.

\footnotetext{
a e-mail : gregory.aiguier@univ-catholille.fr

b e-mail : christelle.didier@univ-lille3.fr
}

This is an Open Access article distributed under the terms of the Creative Commons Attribution License 4.0, which permits unrestricted use, distribution, and reproduction in any medium, provided the original work is properly cited. 
C'est ce contexte et cette évolution du recours à l'éthique qui interpellent les enseignants-chercheurs du Département d'Ethique de l'Institut Catholique de Lille, sollicités pour assurer l'enseignement de l'éthique dans les cursus médicaux, paramédicaux. Bon nombre de questions se posent en effet à eux, qui se posent aussi dans le champ de la formation des ingénieurs. Quel est l'état de l'intégration de l'éthique dans les curriculums de médecine et de l'ingénierie ? Quels projets et quels dispositifs pédagogiques sont proposés ? Qu'en est-il des pratiques effectives de formation (méthodes pédagogiques, modalités d'évaluation, postures pédagogiques) ? Enfin, quelles problématiques spécifiques émergent de ces deux champs ? Mais aussi, qu'est-ce que la comparaison peut apporter à la compréhension des liens existant entre la «professionnalisation » et la formation à l'éthique ?

L'objectif de cette contribution est ainsi d'exposer les premières réflexions théoriques d'une recherche à la croisée de l'éthique et de la pédagogie actuellement menée par ces enseignantschercheurs. Dans un premier temps, elle vise à mieux comprendre l'évolution du recours à l'éthique dans le champ de la médecine et les implications pédagogiques attendues, en appuyant le propos sur la présentation d'un dispositif de formation à l'éthique en médecine. Dans un deuxième temps, il s'agira de mettre en perspective cette approche de l'éthique en médecine avec les pratiques pédagogiques menées dans le milieu des ingénieurs.

Pour conclure, nous soulignerons la nécessité de prolonger cette recherche fondamentale par une démarche de co-élaboration de projets de formation avec les acteurs des pratiques pédagogiques et professionnelles de ces deux champs, et ce dans le souci d'une contextualisation effective des projets pédagogiques et d'une réponse adaptée aux enjeux de formation de ces champs.

\section{L'éthique dans le cursus médical}

\subsection{Evolution du besoin d'éthique en santé : l'éthique comme ressource du professionnalisme}

Les pratiques en santé se sont complexifiées dans le courant du $\mathrm{XX}^{\text {ème }}$ siècle, en devenant une pratique non plus exclusivement technoscientifique, mais aussi une pratique sociale et politique. Plusieurs évolutions significatives en attestent. En tout premier lieu, on peut relever les fortes évolutions liées aux progrès technoscientifiques qui ont eu pour conséquence de générer de nouveaux savoirs, de nouvelles pratiques, et de faire évoluer les prérogatives de la médecine (soin, prévention, prédiction). Ensuite, on peut aussi pointer une certaine forme de socialisation et de politisation des pratiques biomédicales, qui a émergé dans le courant du XX $\mathrm{X}^{\mathrm{ème}}$ siècle, dans le contexte spécifique de l'après Seconde guerre mondiale. En effet, la dénonciation lors du procès de Nuremberg de nombreux scandales liés aux expérimentations sur l'homme, a fait naître une demande de régulation des pratiques biomédicales, mais aussi un besoin de réflexion sur les enjeux de ces dernières. C'est dans ce contexte qu'est apparu le mouvement de la bioéthique, «qui désigne un ensemble de recherches, de discours et de pratiques, généralement pluridisciplinaires, ayant pour objet de clarifier ou de résoudre des questions à portée éthique suscitées par l'avancement et l'application des technosciences biomédicales » (Hottois \& Missa, 2001).

Mais si elles se sont d'abord concentrées sur la régulation des pratiques, les réflexions en bioéthique se sont par la suite recentrées sur le patient (mouvement de l'éthique clinique) avant de s'ouvrir aux dimensions institutionnelles et organisationnelles, et ce dans un contexte économique et social marqué par une raréfaction des ressources et donc par des choix à arbitrer en matière de dépense de santé.

A travers l'évolution interne de la bioéthique, on repère ainsi un double mouvement de la médecine. Celui tout d'abord d'une médecine qui s'interroge sur ses propres pratiques et se nourrit pour ce faire de la réflexion d'autres disciplines, notamment celles des sciences humaines et sociales; et dans le même temps d'une inscription sociale plus forte des pratiques biomédicales, la société entendant influer sur la médecine, sur ses missions et son organisation. 
Cette évolution significative du contexte de la santé a de ce fait contribué à modifier la posture du praticien, dont l'action s'inscrit désormais dans un cadre plus collectif, notamment dans le contexte contemporain des maladies chroniques qui requiert une prise en charge globale intégrant la perspective du patient (Rothier Bautzer, 2002).

C'est en ce sens qu'il faut relire le mouvement de professionnalisation du champ de la santé.

\subsubsection{Une professionnalisation de la médecine}

De fait, jadis perçue comme un art puis comme une discipline scientifique et technique, la médecine se développe aujourd'hui comme une profession (Cruess, Johnston \& Cruess, 2004) socialement instituée, régulée, et dont les compétences s'élargissent.

Les travaux du Collège royal des médecins et chirurgiens du Canada consacrés au projet CanMeds témoignent sur ce point d'un élargissement des compétences des médecins (Frank, 2005) pour permettre à ces derniers de mieux s'insérer dans leur environnement professionnel et de mieux répondre aux besoins de la société ${ }^{3}$.

De manière synthétique, on peut retenir de ces travaux que l'intervention médicale est désormais l'œuvre d'un «professionnelv» reconnu socialement pour son expertise scientifique et technique qui lui donne la capacité de porter un jugement professionnel ; qu'une certaine autonomie (dans le choix des moyens notamment) lui est octroyée compte-tenu de son expertise spécifique ; qu'il lui incombe d'évaluer les finalités de son action compte-tenu des attentes sociales, d'actualiser ses compétences professionnelles dans un souci de qualité optimale, et d'intégrer les aspects socioéconomiques et politiques (injonction de responsabilité). Ensuite, qu'il est enjoint de dépasser les modèles de l'expertise et du paternalisme au profit d'une approche plus coopérative de l'intervention professionnelle (Legault, 2005) centrée sur le patient et intégrant sa perspective, et ce dans la visée d'une prise en charge plus globale du patient. Et enfin qu'une importance est accordée aux activités de recherche et d'enseignement, le médecin se devant de contribuer au développement des connaissances et à la formation des futurs professionnels du champ.

\subsubsection{Emergence d'un professionnalisme réflexif et internalisation de l'éthique}

Dans ce contexte, c'est donc un nouveau type de professionnalisme médical, c'est-à-dire un nouveau type de contrat implicite entre la médecine et la société (Cruess \& Cruess, 2000) qui émerge. Il s'agit en effet de dépasser le seul registre déontologique centré sur les valeurs et les comportements idéaux attendus compte tenu du rôle à exercer, au profit d'une approche plus réflexive (Verkerk, De Bree \& Mourits, 2007), plus critique, intégrant la complexité des pratiques et réinterrogeant le contexte socioprofessionnel dans lequel elles se déploient. Cette évolution du professionnalisme requiert donc de la part des professionnels le développement d'une compétence en éthique leur permettant d'évaluer l'articulation des finalités et des moyens de leur agir, pour ainsi (re)construire de nouvelles formes d'intervention professionnelle plus adaptées.

Dans ce cadre, l'éthique ne se limite plus à viser la compréhension des enjeux moraux inhérents aux pratiques biomédicales. Elle vise plutôt l'engagement des acteurs dans la redéfinition de leur agir professionnel et dans la production, en contexte, des compétences adaptées aux situations problématiques auxquelles ils ont à faire face. Ce recentrement de la formation en éthique sur la capacité d'agir des acteurs s'inscrit dans le tournant pragmatique pris par l'éthique en santé (Aiguier, Le Berre, Vanpee \& Cobbaut, 2012) que l'on peut, avec Marc Maesschalck (2010), caractériser comme le passage d'une éthique axée sur les discours normatifs à propos des pratiques, à une capacité de

\footnotetext{
${ }^{3}$ Les compétences CanMeds : expert médical, communicateur, collaborateur, gestionnaire, promoteur de la santé, érudit, professionnel.
} 
« faire » de l'éthique en situation. Ainsi appréhendée, l'éthique relève d'une ressource intrinsèque que les sujets-professionnels doivent développer pour agir avec compétence, ce terme étant ici défini comme potentialité du sujet à développer un « savoir agir » dans des situations complexes (Tardif, 2006).

Cette nouvelle visée de l'éthique en modifie les pratiques et les dispositifs pédagogiques au sein du cursus médical.

\subsection{Implications pédagogiques de ce tournant pragmatique de l'éthique pour la formation médicale}

Sur le plan des fondements pédagogiques, il ne paraît plus pertinent d'inscrire la formation en éthique dans une logique d'enseignement dans laquelle prévaut une démarche transmissive de savoirs figés dont on ne peut garantir ni le transfert en situation ni l'actualisation. C'est plutôt une logique d'apprentissage inscrite dans la perspective du socioconstructivisme et centrée sur l'apprenant et sur son interaction avec l'environnement comme source d'apprentissage qui semble devoir être privilégiée. Pratiquement, cela se traduit par une articulation plus prononcée entre théorie et pratique et par le recours à des pédagogies actives, réflexives et situationnelles de formation (approches par compétences, démarches de résolution de problèmes). Certains travaux consacrés à la pédagogie de l'éthique en santé rendent compte de cette évolution (Aiguier, Boitte \& Cobbaut, 2012 ; Marcoux \& Patenaude, 2000). Aux modèles déontologiques de la formation à l'éthique visant l'adoption par le professionnel du comportement estimé adéquat par ses pairs, aux modèles « principlistes » visant la déduction par le sujet de principes d'actions, aux modèles plus narratifs et herméneutiques centrés sur le récit du sujet et visant la construction d'une représentation des situations (Fourez, 1994), voire aux modèles centrés sur le développement d'une « sensibilité morale » du sujet (éthique du care) (Gendron, 2009), on privilégie aujourd'hui des modèles de formation plus actifs, réflexifs et situés (Cruess, Cruess \& Steinert, 2009). Ces modèles, centrés sur les aspects socioprofessionnels (Godin, Moulin \& Vanpee, 2004) et sur les acteurs (Molewijk, Abma, Stolper \& Widdershoven, 2008), assimilent l'éthique à une compétence professionnelle, en l'occurrence clinique (Patenaude, Lambert \& Dionne, 2001).

Sur le plan des méthodes pédagogiques, cette compétence en éthique est appelée à se développer à partir d'une démarche d'analyse réflexive, contextuelle mais aussi collective de situations-problèmes (Huber \& Dalongeville, 2011) qui amène les sujets-apprenants à problématiser (Fabre, 2006) l'agir médical pour en repenser les finalités et les moyens.

Mais au-delà d'un ajustement des méthodes pédagogiques, cette approche plus contextuelle et réflexive de la pédagogie de l'éthique questionne son intégration dans le cursus médical.

\subsection{Quelle intégration de l'éthique dans le cursus médical ? L'exemple du programme éthique de la Faculté Libre de Médecine de Lille}

Il ne semble en effet plus pertinent d'organiser un enseignement de l'éthique délié de la pratique clinique. Ce postulat pédagogique inspire l'intervention pédagogique à la Faculté Libre de Médecine (FLM) des enseignants-chercheurs du Centre d'Ethique Médicale (Département d'Ethique de l'Institut Catholique de Lille), responsables des enseignements d'éthique et de sciences humaines et sociales (SHS). Ils proposent en effet un dispositif dont les objectifs généraux visent à permettre aux étudiants de comprendre le contexte contemporain des pratiques en santé, et de se situer comme futurs professionnels d'un champ complexe et en mutation (place du patient-partenaire des soins ; contexte social, politique, culturel et économique; progrès technoscientifiques; etc.).

En première année, c'est dans le cadre de l'enseignement des SHS (Lefeve \& Mino, 2011) structuré autour de six modules (histoire, sociologie, droit, économie, psychologie et philosophie) qu'est abordée l'éthique. Un triple objectif est ici visé : familiariser les étudiants avec les principaux concepts contemporains de chaque discipline (dimension épistémologique) ; partager l'intérêt d'un 
regard croisé de ces disciplines sur ce qu'est la nature et l'évolution de la médecine (dimension pratique); contribuer au développement de leur capacité réflexive en élargissant leur préoccupation à l'ensemble du champ social (dimension citoyenne). Dans la perspective d'une contextualisation de l'apprentissage peu évidente dans une première année lors de laquelle les étudiants n'ont pas de stage, cet enseignement des SHS s'appuie sur une «thématique conductrice », en l'occurrence le cancer (Aiguier, Boury, Oboeuf, Cobbaut, Forzy, Lustman \& Boitte, 2013). Structurer cet enseignement autour du thème du cancer semble pertinent tant du point de vue de la posture pédagogique que de l'approche éthique qui la soustend.

Du point de vue éthique, l'évolution des modalités de prise en charge du cancer met en exergue quatre enjeux essentiels de la médecine contemporaine : (1) le cancer reste une pathologie marquée par une marge d'incertitude que les progrès techniques et scientifiques - s'ils la réduisent - n'abolissent pas ; (2) cette pathologie requiert une prise en charge holistique ; (3) elle génère une transformation de la relation de soin ; (4) la prise en charge du cancer est un lieu d'articulation des préoccupations centrées sur la singularité de l'individu et des préoccupations collectives de santé publique. Du point de vue pédagogique, ce thème comporte un certain nombre de traits qui en favorisent l'appropriation par les étudiants. C'est une pathologie proche et familière (à travers l'expérience des proches et à travers les médias, chacun s'est construit son propre système de représentation du cancer); les éléments de compréhension du contexte semblent à leur portée (beaucoup de ressources - dans toutes les disciplines - sont disponibles pour constituer un parcours pédagogique riche et varié).

En $2^{\text {ème }}$ année (L2), l'enseignement de l'éthique se concentre autour du stage infirmier, première expérience significative des étudiants de médecine. Le débriefing de ce stage infirmier en sous-groupes (15 étudiants) est ainsi l'occasion d'appuyer l'enseignement de l'éthique sur l'expérience vécue par les étudiants ou au moins sur leur observation et/ou ressenti de situations vécues comme déstabilisantes, problématiques, voire parfois inacceptables. Les étudiants sont ainsi mis en situation d'analyse a posteriori des problèmes éthiques issus des pratiques de soins.

En L3, l'enseignement se poursuit par un cours consacré à la subjectivité des étudiants. Un binôme professeur d'éthique et de SHS et un praticien hospitalier assure un cycle de cours centré sur les étudiants considérés comme des sujets éthiques. C'est donc à interpeller leur responsabilité non pas uniquement de soignant mais aussi de citoyen et d'être humain qu'est consacré ce cours.

Fort du premier cycle consacré à une double démarche de contextualisation et de subjectivation, le deuxième cycle des études médicales s'organise autour d'une dizaine d'interventions de $2 \mathrm{~h}$ sur une pratique clinique, et sur un enseignement d'éthique clinique de $24 \mathrm{~h}$. L'enseignement s'appuie sur l'expérience effective des étudiants, et ce dans le cadre d'un apprentissage expérientiel (Albarello, Barbier, Bourgeois \& Durand, 2013) développé dans la perspective du pragmatisme (Dewey, 2011) : il s'agit non pas de faire référence à une expérience, mais d'expérimenter une réflexion éthique collective. On tend ici vers la visée d'une approche plus pragmatique de l'éthique qui rende les étudiants capables d'initier une démarche éthique.

\subsection{Problématiques pédagogiques spécifiques}

Si l'ensemble de ce dispositif vise au final le développement d'une compétence en éthique intégrée à la pratique des médecins, certaines questions se posent.

La première concerne l'évaluation du développement effectif de cette compétence. Sur ce point, s'il semble plus évident d'évaluer l'acquisition d'un certain nombre de ressources de cette compétence (développement d'une capacité critique et réflexive via les exercices rédactionnels, évaluation des comportements et de la sensibilité éthique via des exercices de simulation et de jeux de rôles), l'évaluation du développement d'une réelle compétence - par ailleurs et par définition appelée à se développer tout au long de la vie professionnelle - semble moins aisée. Elle requiert sans doute un renforcement de l'alternance (terrain/école) permettant une évaluation non pas uniquement lors 
des cours à la Faculté, mais sur le terrain. Dans cette perspective, c'est donc une alternance de type intégrative (Mehran, Ronveaux \& Vanhulle, 2007) qui devrait être privilégiée, c'est-à-dire une alternance qui s'articule autour d'un objectif d'apprentissage commun co-construit et co-évalué par les différents partenaires de l'apprentissage (les formateurs, les praticiens et les étudiants). Si les outils d'évaluation existent (portfolio par exemple), c'est la volonté institutionnelle des environnements d'apprentissage (écoles et terrains) qui reste à formaliser.

Quant à la deuxième question, elle porte sur la posture pédagogique des enseignants et plus largement des partenaires de l'apprentissage. Les études médicales peinent encore sans doute à sortir d'une logique transmissive, son rapport à l'expérience pouvant encore parfois se cantonner à une certaine forme de mimétisme. Or la perspective d'une éthique considérée comme dimension intrinsèque de l'agir médical oblige à repenser l'accompagnement pédagogique. Si la posture de l'enseignant transmetteur de ressources reste pertinente lorsqu'il s'agit d'acquérir des savoirs conditionnels et procéduraux nécessaires à l'exercice de la médecine, elle doit aussi s'accompagner d'autres postures : celle du « guide », lorsqu'il s'agit d'accompagner les étudiants pendant leur temps de stage ; celle de l'animateur quand il s'agit de débriefer les expériences vécues par les étudiants en stage. Plus globalement, c'est donc une posture de « compagnon réflexif » (Donnay \& Charlier, 2006) qui semble devoir être privilégiée. Elle implique une capacité à alterner les postures pédagogiques en fonction des séquences pédagogiques. Sa mise en œuvre relève d'une révolution culturelle tant des acteurs que des institutions, qui doivent accepter le glissement vers une perspective socioconstructiviste de l'apprentissage (Vygotski) dans laquelle la relation pédagogique devient moins asymétrique, « celui qui sait » devenant celui qui « accompagne » l'étudiant dans le développement idiosyncrasique de son apprentissage.

\section{La formation éthique dans le champ de l'ingénierie}

\subsection{Une émergence de la question de l'éthique chez les ingénieurs sans demande sociale}

Le développement de la formation à l'éthique des futurs ingénieurs n'est en rien comparable en France à celle concernant les professionnels de la santé. Pourtant le champ de la pratique des ingénieurs a connu, à l'instar de celui des médecins, des transformations majeures, d'où l'intérêt de leur mise en perspective.

L'augmentation des connaissances et des potentialités d'action dont on évoque les impacts sur la médecine a également profondément modifié les milieux industriels, en ouvrant toujours plus le champ des possibles avec ses bienfaits et ses risques de dérives, conduisant à la nécessité de produire une nouvelle « éthique pour la civilisation technologique » (Jonas, 1979). Tandis que la transformation des pathologies a conduit les médecins à travailler avec d'autres types de professionnels, l'évolution des impacts des activités industrielles dans le contexte de la globalisation des échanges, accompagnée de ses impacts sociaux et environnementaux, a conduit les ingénieurs à travailler encore davantage que par le passé dans un contexte caractérisé par sa « complexité socio-technique » (Feltz, Goujon, Hériard Dubreuil, Lavelle \& Lesch, 2007). Le contexte de la seconde guerre mondiale a été un moment de prise de conscience universelle que l'équation « progrès technique égale progrès social » n'était plus à l'ordre du jour. Si les impacts sociaux dramatiques de l'industrialisation avaient déjà bien entamé l'idéologie du progrès, Hiroshima et Nagasaki ont mis les savants en face de l'évidente nécessité de réguler les pratiques scientifiques comme en témoigne le manifeste Russell-Einstein de 1955. En revanche, si l'utilisation, pendant cette même période de crise morale, des savoirs et techniques des ingénieurs au service de l'extermination d'êtres humains a conduit à une remise en question profonde

de la profession en Allemagne, on n'en trouve guère de traces dans d'autres régions du monde (Verein Deutscher Ingenieure, 1950). Contrairement au champ de la recherche biologique et de la santé pour lesquels la seconde guerre mondiale a constitué un jalon fondamental dans la naissance de la bioéthique, 
il n'y a pas eu l'équivalent d'une « techn-éthique » qui serait venue avec un retentissement comparable questionner les sciences appliquées et leurs acteurs centraux que sont les ingénieurs.

Déjà peu nombreux à se préoccuper de la question de la technique - sorte de point aveugle de la pensée depuis l'Antiquité (Goffi, 1988) - les philosophes n'ont pas non plus été d'une grande aide pour aider à penser les enjeux éthiques de la pratique professionnelle des ingénieurs, sauf peut-être en Allemagne, également aux Etats-Unis à partir des années 1980, mais pour des raisons très différentes (Davis, 1998). Bien que la France soit le pays qui a vu se déployer la pensée d'Ellul sur la technique et sur les questions morales que pose son développement dans tous les domaines, et pas seulement ceux de la médecine (Ellul, 1954), bien qu'elle ait été un des premiers pays d'Europe où une recherche en éthique de l'ingénierie ait été menée dès le début des années 1990 (Hériard Dubreuil, 1997 ; Didier, GireauxGeneau \& Hériard Dubreuil, 1998), la place de l'éthique dans la profession et dans les formations d'ingénieur y est demeurée jusqu'à aujourd'hui extrêmement marginale.

Si on ne peut pas dire que la question éthique n'a absolument pas croisé le monde des ingénieurs, ni celui de leur formation en France, force est de constater qu'il n'existe pas de la part des professionnels une demande d'éthique, comparable à celle émanant des milieux de la santé, pas plus que de la part de la société en générale.

En ce qui concerne les milieux professionnels, un intérêt pour la question a émergé en France au cours des années 1990, dont témoigne la rédaction du premier « code de déontologie », ou plutôt de l'adaptation au contexte français du « code de conduite » proposé par la Fédération Européenne des Associations Nationale d'ingénieurs. Ce texte publié en 1996 par le Conseil national des ingénieurs et scientifiques de France (CNISF) a été très peu diffusé dans les milieux professionnels et semble indirectement connu des ingénieurs membres du CNISF, via leur association d'anciens élèves. En 2001, un nouveau texte, appelé «Charte d'éthique de l'ingénieur » a remplacé le précédent. Davantage diffusé sur les sites internet des écoles d'ingénieurs, il reste peu connu des professionnels et des étudiants qui sont très rarement invités à les étudier - ou tout simplement à en prendre connaissance - au cours de leur formation.

En Allemagne, ce sont les actes dramatiques posés pendant la seconde guerre mondiale par des ingénieurs qui les ont conduit à se remettre en question. Un demi-siècle après avoir publié solennellement une confession quasi religieuse affirmant solennellement les obligations morales de la profession, l'association des ingénieurs allemands a produit un texte (qui n'est pas un code) visant à proposer un cadre pour la réflexion éthique de ses membres (Didier, 2007). Au Royaume-Uni, les ingénieurs qui avaient pourtant été les premiers au monde à promulguer un code de conduite professionnelle ont attendu près d'un siècle pour publier un second texte du genre (Didier, 2008). Mais comme pour le texte publié par la VDI en 2002, il ne s'agit pas au sujet du texte britannique d'un code de déontologie, tel qu'il en existe en France pour diverses professions libérales dont la médecine, ou pour un nombre plus important d'activités au Québec. Il s'agit plutôt d'une réflexion élaborée collectivement au sein d'un organisme représentatif des ingénieurs au sujet des enjeux éthiques liés à leur activité professionnelle. En France, ni le premier code de déontologie ni la charte d'éthique qui l'a remplacée ne sont en revanche accompagnés d'une réflexion permettant de situer ce document dans le contexte de son écriture, ou d'en éclairer le sens pour les premiers concernés. Un bref préambule rappelle simplement la prise de conscience par la profession des impacts importants des techniques sur la société, «avancées » qui sont «porteuses du risque de fortes nuisances ».

\subsection{Des expériences pédagogiques éclatées dans un contexte de formation peu facilitant}

Il n'y a donc en matière de demande d'éthique concernant les ingénieurs, en France, que des signaux faibles. Tout au plus l'éthique est-elle perçue comme un «supplément d'âme » non intégré au métier. La demande sociale de régulation des pratiques industrielles ne s'adresse pas aux ingénieurs mais 
aux organisations qui sont leurs principaux employeurs : les grandes entreprises industrielles privées capitalistiques. Cette demande se traduit par une importante littérature où le souci de l'éthique dans un monde industriel qui change et appelle de nouvelles formes de régulation s'appelle "Responsabilité sociale des entreprises » (RSE) ou « responsabilité sociale des organisations » (RSO) (Postel, Cazal, Chavy \& Sobel, 2011).

En ce qui concerne les formations, il serait injuste de dire qu'il n'existe en France aucun cours destiné à des futurs ingénieurs ou aucune proposition pédagogique abordant les questions éthiques liées à leur profession. Des enseignants ont mené depuis des années des expériences diverses en ce domaine. Mais combien sont-ils ? Où sont-ils ? Quels sont les statuts des intervenants en ce domaine ? Qu'enseignent-ils exactement dans un cours d'éthique ? Quels supports pédagogiques mobilisent-ils et quelles méthodes ? Se connaissent-ils ? Où et comment confrontent-ils leur méthode et discutent-ils de leurs pratiques ? Quels progrès dans une pédagogie de l'éthique pour les ingénieurs ont été accomplis ?

Force est de constater qu'il est particulièrement difficile de répondre à ces questions et que les traces de cette réalité pédagogique indéniable sont également faibles et surtout difficiles à analyser. Les raisons en sont diverses : l'une d'entre elles est que le concept même d' "éthique de l'ingénieur », ou d' « éthique de l'ingénierie » est loin d'aller de soi dans les milieux de la formation des ingénieurs, pas plus que dans la profession d'ailleurs, pas plus non plus que dans les milieux industriels employeurs principaux des futurs diplômés, au point que l'on peut se demander parfois ce qui a pu motiver de telles expériences.

Aux Etats-Unis, la formation des ingénieurs à l'éthique professionnelle est arrivée des années après l'émergence d'une déontologie (non dotée de pouvoir de sanction), née dans un contexte de « professionnalisation » (dans l'acception de ce terme tout à fait particulière dans la sociologie des professions anglo-saxonnes (Hériard Dubreuil \& Didier, 2008). Au Québec, une formation à la déontologie professionnelle a existé depuis qu'une déontologie ayant force de loi a été promulguée par l'Ordre des Ingénieurs du Québec et que les examens professionnels ont compris des épreuves sur ces sujets (Didier, 2009). Si des formations à l'éthique - et pas seulement à la déontologie - existent, elles y sont moins courantes qu'aux Etats-Unis. En effet il n'y a que dans le cadre de la préparation à l'examen permettant de devenir un Professional Engineer (PE) qu'il existe aux Etats-Unis des épreuves visant à vérifier la connaissance des normes déontologiques et cela ne concerne que $10 \%$ des diplômés. Il n'en reste pas moins que la diversité des approches proposées dans les formations d'ingénieurs au Québec - ajoutée au fait que des recherches académiques prenant comme objet la formation éthique dans des professions autres que médicales existent - a rendu possible dès le début des années 2000 une mise en perspective comparée critique (Legault, 2007).

Dans le cas du Québec comme dans celui des Etats-Unis, il est à noter que les formations à l'éthique se sont développées dans un contexte où préexistait de façon officielle un ensemble de normes visant à réguler les pratiques professionnelles, soit de façon contraignante pour les ingénieurs québécois, soit de façon non contraignante et liée à l'adhésion volontaire à une association professionnelle, par ailleurs et très courante, pour les américains. Dans le cas des Etats-Unis, il convient de noter l'influence du soutien de la puissance National Science Foundation dès le début des années 1980 à la création de manuels d'éthique de l'ingénierie, et de l'exigence inscrite dans ses critères à partir des années 2000 par l'Accreditation Board of Engineering and Technology (ABET) sur le développement de propositions de formation en éthique pour les futurs ingénieurs (Didier, 2009). Avant la demande sociale, c'est une demande émanant des milieux académiques qui a dominé le développement de la formation éthique des ingénieurs outre Atlantique. Pourtant d'autres modèles sont possibles comme l'illustre le cas des Pays-Bas où c'est une volonté politique qui a soutenu le développement de l'éthique dans la formation des futurs ingénieurs et où la création de cours d'éthique n'a eu que peu de rapport avec la préexistence d'une déontologie professionnelle (Van de Poel, Zandvoort \& Brumsen, 2001).

Dans d'autres pays d'Europe, des cours ont été créés soit par effet de mimétisme par rapport à l'existence de formation à l'éthique dans d'autres domaines (médecine, travail social...), soit sous l'impulsion d'individus ayant connu l'existence de cours d'éthique pour ingénieurs dans d'autres pays, 
soit sous l'impulsion d'une association professionnelle (pas forcément dotée d'une déontologie), soit à la demande d'un agent prescripteur (ministère de l'éducation ou organisme d'habilitation des diplômes ou des formations). Dans le cas de la France, l'émergence de cours d'éthique dans les formations des ingénieurs a bénéficié dans certaines écoles d'un soutien local, lié le plus souvent à l'initiative d'un enseignant ou d'un acteur en position de décision personnellement motivé à développer ce champ. Elle a aussi bénéficié de la publication depuis 1996 de la recommandation à introduire « de l'éthique » dans les formations, formulée par la Commission des Titres d'ingénieurs, organisme d'accréditation considéré comme la pièce centrale du système de formation des ingénieurs français depuis sa création en 1934. Mais là encore, si la demande existe, elle brille par son opacité. Elle est même devenue de plus en plus floue au fur et à mesure de ses reformulations, ou en tout cas éloignée de ce que l'on aurait pu attendre d'une demande de formation à l'éthique professionnelle pour des futurs ingénieurs (Didier, 2007).

Il est guère étonnant que les approches pédagogiques de l'éthique dans la formation des ingénieurs soient aussi diverses que le sont les intervenants, vacataires de passage, experts autoproclamés de l'éthique dans certain cas. Etant donné le faible nombre d'heures consacrées à ce thème, l'objectif atteignable consiste le plus souvent à « sensibiliser » les étudiants aux enjeux éthiques des techniques pas forcément à ceux de leur profession d'ailleurs-. Dans le meilleur des cas, on peut espérer leur faire prendre conscience de la pertinence qu'il peut y avoir à mener une réflexion de type éthique sur le champ d'activité professionnelle auquel ils se préparent. Mais cette pertinence n'est pas toujours partagée ni par les prescripteurs, ni par les futurs employeurs. Si le green-washing peut être un thème intéressant et pertinent à aborder dans un cours d'éthique destiné à de futurs ingénieurs, on ne manquera pas de penser que certains cours d'éthique sont des alibis qui s'apparentent à une pratique comparable.

\section{Conclusion}

Les réflexions précédentes font état d'une disparité d'approche de l'éthique, de pratiques pédagogiques et de problématiques afférentes différentes selon les champs étudiés.

Pour une équipe d'enseignants et de chercheurs en éthique devant répondre à des demandes de formation très hétéroclites, voire parfois confuses, il semble ainsi vain de vouloir absolument homogénéiser les pratiques d'intervention pédagogique. L'enjeu semble plutôt de se rendre attentif aux contextes singuliers dans lesquels ils sont amenés à intervenir. Autrement dit, face à la tentation de réponses pédagogiques universelles, prédéfinies, et de constitution d'une boîte à outils standardisée, il s'agit plutôt de réfléchir aux modalités d'accompagnement des acteurs et/ou des écoles dans la définition de leur besoin et dans la construction des réponses adaptées.

Pour l'équipe pédagogique du Département d'Ethique, l'expertise attendue porte ainsi sur sa capacité d'accompagner les acteurs et les institutions dans cette démarche.

L'organisation d'une journée d'étude consacrée à la formation à l'éthique ${ }^{4}$ a été sur ce point une première entreprise. Elle visait à réunir des formateurs, des responsables pédagogiques et des directeurs d'écoles, mais aussi des chercheurs et des professionnels issus de divers horizons, pour réfléchir collectivement aux problématiques inhérentes à la formation en éthique, sur base d'expériences partagées.

Renonçant à la posture d'expert et d'évaluateur des pratiques de formation en éthique que l'on voudrait parfois leur prêter, les enseignants et chercheurs du Département d'Ethique s'engagent ainsi davantage dans une co-élaboration pédagogique, respectueuse des acteurs, des contextes singuliers, de leur histoire, de leur culture, et qui relève finalement d'une véritable éthique de l'accompagnement (Beauvais, 2007).

\footnotetext{
4 Journée d'étude du Département d'Ethique organisée le vendredi 24 mai 2013.
} 


\section{Références}

[1] Aiguier, G., Boitte, P. \& Cobbaut, J.-Ph. (2012). Repenser la formation à l'éthique au prisme du pragmatisme : fondements et implications pédagogiques.Ethica Clinica, 68,16-23.

[2] Aiguier, G., Boury, D., Oboeuf, A., Cobbaut, JP, Forzy, G., Lustman, M. \& Boitte, P. (2013). Ethique et cancer : fil rouge dans une formation initiale de sciences humaines et sociales en médecine (PACES). Psycho-oncologie, 7, 97-105.

[3] Aiguier, G., Le Berre, R., Vanpee, D. \& Cobbaut, J.-Ph. (2012). Du pragmatique au pragmatisme : quels enjeux pour la formation à l'éthique ? Journal International de Bioéthique, 23(4), 123-148.

[4] Albarello, L., Barbier, J.-M., Bourgeois, E. \& Durand, M. (2013). Expérience, activité, apprentissage. Paris : Presses universitaires de France.

[5] Beauvais, M. (2004). Des principes éthiques pour une philosophie de l'accompagnement. Savoirs, revue internationale de recherches en éducation et formation des adultes, 3(6), 99-113.

[6] Cruess, R. L. \& Cruess S. R. (2000). Professionalism : a contract between medicine and society. CMAJ, 162(5), 668-669.

[7] Cruess, R. L., Cruess, S. R. \& Steinert, Y. (2009). Teaching Medical Professionnalism. New York : Cambridge University Press

[8] Cruess, S. R., Johnston, S. \& Cruess, R. L. (2004). "Profession": A working definition for medical educators. Teaching and Learning in Medicine, 16, 74-6.

[9] Davis, M. (1998). Thinking like an Engineer, Study in the Ethics of a Profession.Oxford: Oxford University Press.

[10] Dewey, J. (2011). Expérience et éducation. Paris: Armand Colin

[11] Didier, C. (2007). Quels contenus pour un enseignement en éthique pour de futurs ingénieurs ? Bilan après 10 ans d'expérience d'enseignement. In B. Feltz B., P. Goujon, B. Hériard Dubreuil, S. Lavelle \& W. Lesch (Ed.), Ethique, complexité et démocratie (pp. 271-287). Louvain-la-Neuve : Academia-Bruylant.

[12] Didier, C. (2008). Penser l'éthique des ingénieurs. Paris : PUF.

[13] Didier, C. (2009). Les ingénieurs et l'éthique professionnelle : pour une approche comparative de la déontologie. In C. Demazière \& C. Gadéa, Sociologie des groupes professionnels. Acquis récent et nouveaux défis (pp. 208-2018). Paris: La découverte.

[14] Didier, C., Gireaux-Geneau, A. \& Hériard Dubreuil, B. (Ed.). (1998). Ethique industrielle. Textes pour un débat. Bruxelles: De Boeck.

[15] Donnay, J., Charlier, E. (2006). Apprendre par l'analyse de pratiques : Initiation au compagnonnage réflexif. Namur: Presses universitaires de Namur

[16] Ellul, J. (1954). La technique ou l'enjeu du siècle. Paris: Armand Colin.

[17] Fabre, M. (2006). Situations de formation et problématisation. Bruxelles : De Boeck.

[18] Feltz, B., Goujon, P., Hériard Dubreuil, B., Lavelle \& S., Lesch, W. (Ed.) (2007). Ethique, complexité et démocratie, Louvain-la-neuve : Academia-Bruylant,

[19] Fourez, G. (1994). Constructivisme et justification éthique. Revue des sciences de l'éducation, 20(1), 157-174

[20] Frank, J. R. (Ed.). (2005). Le cadre des compétences des médecins CanMEDS 2005. De meilleures normes. De meilleurs médecins. De meilleurs soins. Ottawa : Le Collège royal des médecins et chirurgiens du Canada.

[21] Gendron, C. (2009). Développer la compétence éthique par le biais de l'éthique du care : Une utopie ? In F. Jutras \& C. Gohier. Repères pour l'éthique professionnelle des enseignants. SainteFoy: Presses universitaires du Québec.

[22] Godin, V., Moulin, D. \& Vanpee, D. (2004). La formation aux enjeux sociaux et éthiques de la santé et de la médecine : un défi ? L'expérience de la faculté de médecine de l'université catholique de Louvain, Pédagogie Médicale, 5(1),35-42 
Les sciences humaines dans les parcours scientifiques et techniques professionnalisants

[23] Goffi, J.-Y. (1988). La philosophie de la technique. Paris: Presses universitaires de France.

[24] Hériard Dubreuil B. (1997). Imaginaire technique et éthique sociale. Essai sur le métier d'ingénieur. Bruxelles : De Boeck.

[25] Hériard Dubreuil, B. \& Didier, C. (2008). "Engineering Ethics: Europe”. In C. Mitcham, The encyclopedia of Science, Technology and Ethics (pp. 632-635). New-York: Mac Millan,.

[26] Hottois, G. \& Missa, J.-N. (Ed.). (2001). Nouvelle encyclopédie de bioéthique. Bruxelles: De Boeck.

[27] Huber, M. \& Dalongeville, A. (2011). (Se) former par les situations-problèmes : Des déstabilisations constructives. Lyon : Chronique sociale.

[28] Jonas, H. (1979/1998), Le principe responsabilité. Une éthique pour la civilisation technologique, Paris: Flammarion.

[29] Lefeve, C., Mino, J.-C. (2011). Former de vrais thérapeutes. La place des sciences humaines et sociales dans les études de médecine. Etudes 4142, 187-198.

[30] Legault, G. A. (2004). Professionnalisme et délibération éthique : manuel d'aide à la décision responsable. Sainte-Foy: PUQ.

[31] Legault, G. A. (2007). Ethique et complexité dans le domaine du génie : quelle formation humaniste retenir ? Bilan de l'expérience québécoise de formation humaniste des ingénieurs. In B. Feltz B., P. Goujon, B. Hériard Dubreuil, S. Lavelle \& W. Lesch (Ed.), Ethique, complexité et démocratie (pp.237-252). Louvain-la-neuve: Academia-Bruylant.

[32] Legault, G. A. (Ed.). (2005). Crise d'identité professionnelle et professionnalisme, Saint-Foy: Presses universitaires du Québec.

[33] Maesschalck, M. (2010). Transformations de l'éthique : de la phénoménologie radicale au pragmatisme social. Buxelles: Peter Lang.

[34] Marcoux, H. \& Patenaude, J. (2000). L'éthique dans la formation médicale. Où en sommesnous ? Où allons-nous ? Pédagogie Médicale, 1, 23-30.

[35] Mehran, F., Ronveaux, C. \& Vanhulle S. (éds) (2007). Alternances en formation. Bruxelles : De Boeck.

[36] Molewijk, A. C., Abma, T., Stolper M., \& Widdershoven, G. (2008). Teaching ethics in the clinic. The theory and practice of moral case deliberation. J. Med. Ethics, 34, 120-124

[37] Patenaude, J., Lambert, C., Dionne, M. \& al. (2001). L'éthique comme compétence clinique : modélisation d'une approche dialogique, Pédagogie Médicale, 2(2), 71-80.

[38] Postel, N., Cazal, D., Chavy, F. \& Sobel, R. (Ed.). (2011). La RSE, une nouvelle régulation du capitalisme. Villeneuve d'Ascq : Presses Universitaires du Septentrion.

[39] Rothier Bautzer, E. (2002). Pratiques soignantes en mutation : de la lutte contre la maladie à la collaboration avec le patient. Revue française de pédagogie, 138, 39-50.

[40] Tardif, J. (2006). Evaluation des compétences : documenter le parcours de développement. Chenelière Education

[41] Van de Poel, I.R., Zandvoort, H. \& Brumsen, M. (2001). Ethics and engineering courses at Delft University of Technology: Contents, educational setup and experiences. Science and Engineering Ethics 7(2), 267-282.

[42] Verein Deutscher Ingenieure .(1950). Bekenntnis des Ingenieurs (La profession des ingénieurs).

[43] Verkerk, M. A., De Bree, M. J. \& Mourits, M. J. E. (2007). Reflective professionalism: interpreting CanMEDS' "professionalism”. Journal of Medical Ethics, 33(11), 663-666. 